\title{
Competition between protons and substrate for binding to the major facilitator superfamily multidrug/ $\mathrm{H}^{+}$ antiporter MdtM
}

\author{
Christopher J. Law * (D) \\ School of Biological Sciences, Queen's University Belfast, Belfast, United Kingdom \\ ${ }^{\star}$ Corresponding author. Email: c.law@qub.ac.uk
}

(Received 18 August 2021; Revised 29 October 2021; Accepted 30 October 2021)

\begin{abstract}
Proton electrochemical gradient-driven multidrug efflux activity of representatives of the major facilitator superfamily (MFS) of secondary active transporters contributes to antimicrobial resistance of pathogenic bacteria. Integral to the mechanism of these transporters is a proposed competition between substrate and protons for the binding site of the protein. The current work investigated the competition between protons and antimicrobial substrate for binding to the Escherichia coli MFS multidrug/ $\mathrm{H}^{+}$antiporter MdtM by measuring the quench of intrinsic protein fluorescence upon titration of substrate tetraphenylphosphonium into a solution of purified MdtM over a range of $\mathrm{pH}$ values between $\mathrm{pH} 8.8$ and 5.9. The results, which revealed that protons inhibit binding of substrate to MdtM in a competitive manner, are consistent with those reported in a study on the related MFS multidrug/ $\mathrm{H}^{+}$antiporter MdfA and provide further evidence that competition for binding between substrate and protons is a general feature of secondary multidrug efflux.
\end{abstract}

Key words: antimicrobial resistance; intrinsic fluorescence quenching; membrane transporter; substrate binding

\section{Introduction}

Antimicrobial resistance of pathogenic bacteria represents a global public health threat and the activities of secondary active membrane transporters that are members of the major facilitator superfamily (MFS) contribute to this phenomenon. These transporters utilize the transmembrane electrochemical proton gradient to drive antimicrobials out of the cell (Fluman \& Bibi, 2009). Biochemical and structural data for the bacterial MFS multidrug/ $\mathrm{H}^{+}$antiporters are consistent with a general mechanism that constrains the simultaneous binding of substrate and protons to the protein to enable the loading, transmembrane transport, and subsequent periplasmic release of the cytotoxic cargo, while at the same time, impeding any proton leakage that could injure or kill the cell (Zhang et al., 2015). The feasibility of such a mechanism is dependent on the competition between substrate and protons for binding to the transporter (Schuldiner, 2014), which in turn is mediated by tuning of the $\mathrm{p} K_{\mathrm{a}}$ of the carboxyl groups of one or more highly conserved acidic residues located within or near the substrate binding site in the membrane-embedded regions of the protein (Adler et al., 2004; Sigal et al., 2006). A previous study of the model Escherichia coli MFS multidrug efflux antiporter MdfA demonstrated that substrates and protons compete for binding to 
the protein (Fluman et al., 2012). The present study investigated the effects of proton concentration on binding of antimicrobial substrate tetraphenylphosphonium $\left(\mathrm{TPP}^{+}\right)$to the E. coli MFS multidrug/ $\mathrm{H}^{+}$ antiporter MdtM and provides additional supporting evidence that the competition for binding between drug substrate and protons is a general feature of secondary multidrug efflux.

\section{Methods}

\subsection{Plasmids}

Design and construction of the expression plasmid that contained the 1,230 bp coding region of the $m d t M$ open reading frame has been described in detail before (Holdsworth \& Law, 2012). Briefly, the construct encoded the 410 amino acid residues of MdtM with a C-terminal myc-epitope and a hexahistidine tag to facilitate purification of the protein. Inclusion of a thrombin-specific proteolysis site permitted cleavage of the $m y c$-His tag.

\subsection{Overexpression and purification of $M d t M$}

MdtM was overexpressed in E. coli LMG194 cells and purified using a previously described protocol (Alegre \& Law, 2015).

\subsection{Substrate binding assays}

The affinity of purified MdtM in $20 \mathrm{mM}$ Bis-Tris propane (titrated to the appropriate $\mathrm{pH}$ with $\mathrm{HCl}$ ), $100 \mathrm{mM} \mathrm{NaCl}, 10 \%(\mathrm{v} / \mathrm{v})$ glycerol, and $0.1 \%(\mathrm{w} / \mathrm{v}) n$-dodecyl $\beta$-D-maltoside (DDM) detergent for TPP ${ }^{+}$ substrate was determined over a $\mathrm{pH}$ range of $5.9-8.8$ by intrinsic tryptophan fluorescence quenching studies. Steady state fluorescence measurements were performed on sample in a $1.0 \mathrm{~cm} \times 0.5 \mathrm{~cm}$ quartz cuvette using a Fluoromax-4 fluorometer (Horiba, UK) equipped with a magnetically stirred and temperature-controlled cuvette holder set to $25^{\circ} \mathrm{C}$. The longer pathlength of the cuvette was oriented toward the excitation source. Excitation and emission wavelengths were set to $295 \mathrm{~nm}$ (to excite tryptophans of MdtM exclusively) and $335 \mathrm{~nm}$ (the maximum fluorescence emission wavelength of MdtM), respectively. Excitation slit width was set to $1.5 \mathrm{~nm}$ and emission slit width to $3.0 \mathrm{~nm}$.

Freshly purified MdtM was added to the cuvette to a final concentration of $0.22 \mu \mathrm{M}$ in $1.5 \mathrm{ml}$ and allowed to equilibrate to $25^{\circ} \mathrm{C}$ for $5 \mathrm{~min}$. The protein solution was then titrated with $\mathrm{TPP}^{+}$by the sequential addition of a stock $\mathrm{TPP}^{+}$solution to a final concentration of $100 \mu \mathrm{M}$. A 60 s equilibration period between addition of $\mathrm{TPP}^{+}$and measurement of fluorescence emission was allowed. In all the fluorescence experiments, the total volume of stock $\mathrm{TPP}^{+}$solution added to the protein sample was less than $2 \%$ of the initial assay volume. Measurements were performed in triplicate and to ensure consistency and reproducibility of the titrations, the same stocks of $\mathrm{TPP}^{+}$and the same set of calibrated autopipettes (Gilson, UK) were used for all the substrate binding experiments. The collected buffer subtracted data were corrected for dilution and used to calculate a percentage fluorescence quench for each TPP addition. Due to the negligible UV absorbance of $\mathrm{TPP}^{+}$at the fluorescence excitation wavelength of $295 \mathrm{~nm}$, correction for inner filter effects was not required. The resulting intrinsic fluorescence quenching curves were analyzed using nonlinear regression binding analysis available in GraphPad Prism v 9.1.0 (GraphPad Software Inc., San Diego, CA). This approach permitted extraction of the apparent equilibrium dissociation constant, $K_{d}^{\text {app }}$, of binding of $\mathrm{TPP}^{+}$to MdtM at each $\mathrm{pH}$ tested using the equation $y=B_{\max }\left[\mathrm{TPP}^{+}\right] /\left(K_{d}+\left[\mathrm{TPP}^{+}\right]\right)$.

\section{Results}

Competition between protons and antimicrobial substrate for binding MdtM was studied by measuring the quench of intrinsic protein fluorescence upon titration of $\mathrm{TPP}^{+}$into a solution of purified MdtM at a 
range of different $\mathrm{pH}$ values (Figure 1). Increasing the proton concentration by acidification of the protein solution reduced the affinity of MdtM for $\mathrm{TPP}^{+}$substrate (Figure 2), suggesting that protons inhibit binding of substrate in a competitive manner. The $K_{d}^{\text {app }}$ measurements of $\sim 400 \mathrm{nM}$ for TPP binding to MdtM at the neutral and alkaline $\mathrm{pH}$ values tested compare well with a previously published $K_{d}^{\text {app }}$ for $\mathrm{TPP}^{+}$binding to the protein at $\mathrm{pH} 8.0$ (Alegre et al., 2016). A fit of the binding data in Figure 2 enabled the analysis of the effects of proton concentration on $\mathrm{TPP}^{+}$binding affinity. This analysis estimated an inhibition constant, $K_{\mathrm{i}}$, of $0.6 \mu \mathrm{M}$ for protons, which corresponded to a $p K_{a}$ of 6.23 . To ensure that the binding data were not compromised by changes in the structural integrity of solubilized MdtM at the extremes of $\mathrm{pH}$ tested, the fluorescence emission spectrum of the protein was measured between 310 and $400 \mathrm{~nm}$ at $\mathrm{pH}$ values of 5.9 and 8.8 (Figure 3). These spectra were compared to the spectrum of MdtM denatured by incubation at $\mathrm{pH} 8.0$ in a $1 \%(\mathrm{v} / \mathrm{v})$ sodium dodecylsulphate (SDS) solution (Figure 3). Denaturation by SDS resulted in a significant reduction of the fluorescence intensity and a $\sim 8 \mathrm{~nm}$ red-shift of the maximum fluorescence emission peak of MdtM compared to the spectra
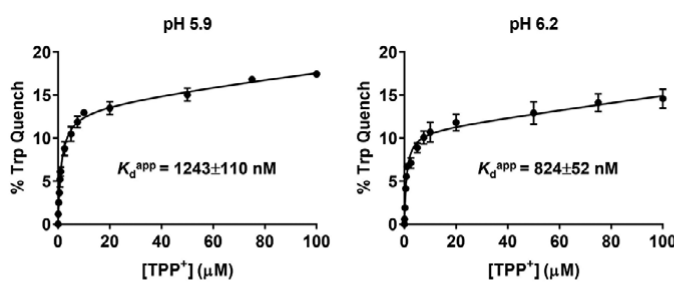

$\mathrm{pH} 7.0$
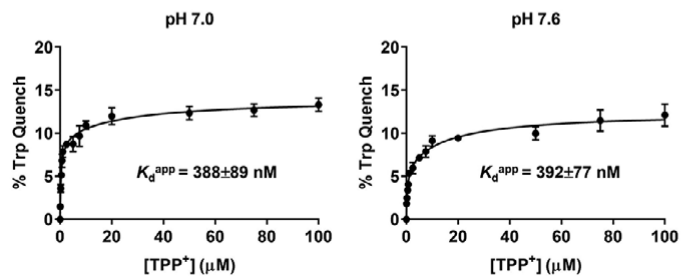

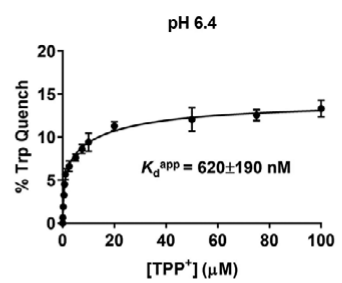

pH 8.3

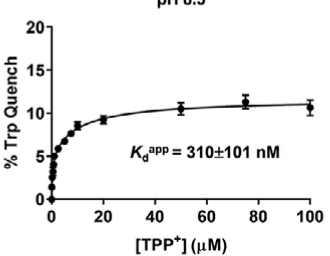

pH 6.7

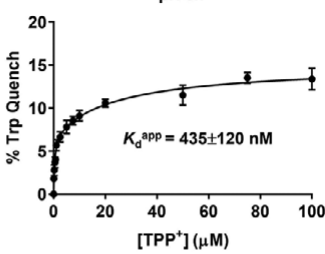

pH 8.8

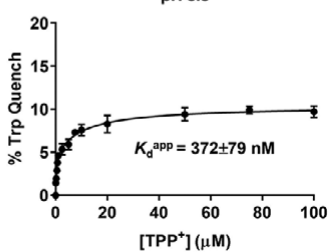

Figure 1. Saturation curves for $\mathrm{TPP}^{+}$substrate binding to MdtM in DDM detergent solution at different pH values. Binding was measured by concentration-dependent quenching of MdtM intrinsic fluorescence emission at $335 \mathrm{~nm}$. Data points and error bars represent the mean and SEM, respectively $(n=3)$. Data were fitted to a binding equation using nonlinear regression (solid line) to enable extraction of apparent dissociation constant, $K_{d}{ }^{\text {app }}$, values.

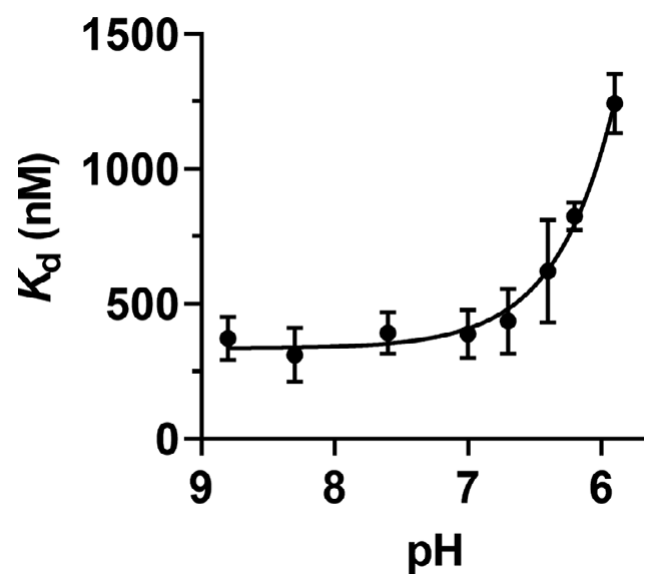

Figure 2. Affinity (as represented by the apparent dissociation constants reported in Figure 1) of purified, detergentsolubilized MdtM for substrate $\mathrm{TPP}^{+}$as a function of proton concentration (represented as $\mathrm{pH}$ ). Data points and error bars represent the mean and SEM, respectively $(n=3)$. The data fitted to a previously published equation (Fluman et al., 2012) that describes competitive binding between $\mathrm{TPP}^{+}$and protons. 


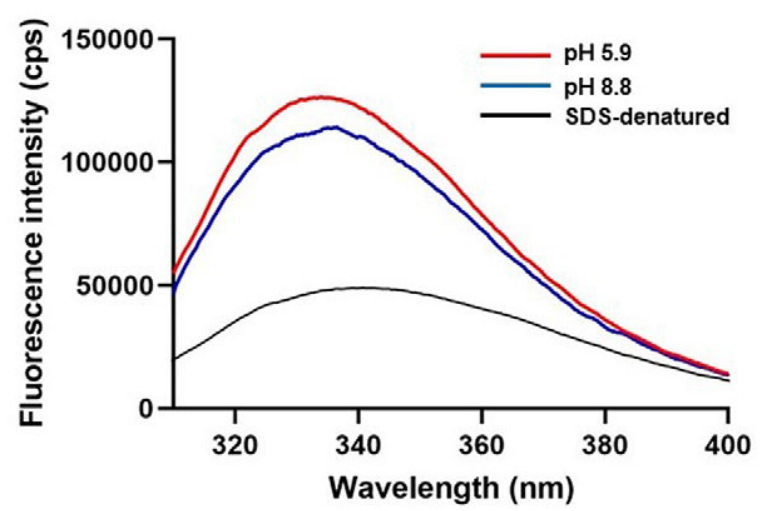

Figure 3. Fluorescence emission spectra of purified MdtM in DDM detergent solution at $\mathrm{pH} 6.2$ and 8.8 , and $\mathrm{MdtM}$ at $\mathrm{pH} 8.0$ denatured in $1 \%$ SDS.

obtained for nondenatured protein. These data support that the integrity of MdtM was not affected by acidification or alkalinization of the protein solution.

\section{Discussion}

The mechanism of secondary MFS drug/ $\mathrm{H}^{+}$antiporters suggests that different binding affinities for the drug cargo must exist with high affinity binding to the protein in the "inward-facing" conformation to scavenge drugs from the cell interior and low affinity binding in the "outward-facing" conformation to enable the drug to be dispensed into the periplasm or extracellular milieu. Such transmembrane movement of drug substrate is coupled to the counter-movement of protons across the membrane via a ping-pong mechanism, in which the substrate must be released prior to binding and subsequent translocation of the counterion (Law et al., 2008). Competition between protons and substrate is regarded as integral to the catalytic transport activity of the electrochemical proton gradient-driven MFS antiporters. In the MFS drug/ $\mathrm{H}^{+}$antiporter MdfA, the whole process is modulated by protonation of two conserved, membrane-embedded acidic residues; a glutamate at position 26 and aspartate at position 34 (Fluman et al., 2012). MdtM possesses two membrane-embedded aspartate residues at positions 22 and 30 (Holdsworth \& Law, 2012) and it is pertinent to speculate that these residues represent the protonation sites in that transporter.

The nature of the competition between substrate and protons can vary between MFS family members. In MdfA, this competition is allosteric with protons and $\mathrm{TPP}^{+}$binding to different sites in the protein (Fluman et al., 2012). It is likely that the same mutually exclusive binding of protons and substrate is a feature of MdtM. In contrast, the MFS multidrug transporter LmrP from Lactococcus lactis exploits a combination of direct and indirect competition for its function (Schaedler \& van Veen, 2010). Irrespective of the exact flavor of competition proposed for different secondary multidrug family members of the MFS, the inhibition of $\mathrm{TPP}^{+}$binding to MdtM by protons demonstrated by the current work is a further indication that substrate/counterion competition is a feature of MFS antiporters in general.

\section{Conclusion}

The work presented here on the MFS drug $/ \mathrm{H}^{+}$antiporter MdtM validates a previous study on the related MdfA protein and provides additional strong empirical evidence that interplay between protons and substrate during binding to MFS drug/ $\mathrm{H}^{+}$antiporters is common to these proteins, and essential to their function.

Funding statement. This work was funded in part by BBSRC grant BB/K014226/1 to C.J.L. 
Conflict of interest. The author declares none.

Authorship contributions. C.J.L. conceived the work, performed the experiments, interpreted the data, produced the figures, and wrote the manuscript.

Data availability statement. Data used for this article are available from the author on reasonable request.

Conflicts of interest. none.

\section{References}

Adler, J., Lewinson, O., \& Bibi, E. (2004). Role of a conserved membrane-embedded acidic residue in the multidrug transporter MdfA. Biochemistry, 43, 518-525. https://doi.org/10.1021/bi035485t

Alegre, K. O., \& Law, C. J. (2015). Purification of a multidrug resistance transporter for crystallization studies. Antibiotics, 4, 113-135. https://doi.org/10.3390/antibiotics4010113

Alegre, K. O., Paul, S., Labarbuta, P., \& Law, C. J. (2016). Insight into determinants of substrate binding and transport in a multidrug efflux protein. Scientific Reports, 6, 22833. https://doi.org/10.1038/srep22833

Fluman, N., \& Bibi, E. (2009). Bacterial multidrug transport through the lens of the major facilitator superfamily. Biochimica et Biophysica Acta, 1794, 738-747. https://doi.org/10.1016/j.bbapap.2008.11.020

Fluman, N., Ryan, C. M., Whitelegge, J. P., \& Bibi, E. (2012). Dissection of mechanistic principles of a secondary multidrug efflux protein. Molecular Cell, 47, 777-787. https://doi.org/10.1016/j.molcel.2012.06.018

Holdsworth, S. R., \& Law, C. J. (2012). Functional and biochemical characterisation of the Escherichia coli major facilitator superfamily multidrug transporter MdtM. Biochimie, 94, 1334-1346. https://doi.org/10.1016/j.biochi.2012.03.001

Law, C. J., Maloney, P. C., \& Wang, D. N. (2008). Ins and outs of major facilitator superfamily antiporters. Annual Review of Microbiology, 62, 289-305. https://doi.org/10.1146/annurev.micro.61.080706.093329

Schaedler, T. A., \& van Veen, H. W. (2010). A flexible cation binding site in the multidrug major facilitator superfamily transporter LmrP is associated with variable proton coupling. The FASEB Journal, 24, 3653-3661. https://doi.org/10.1096/ fj.10-156927

Schuldiner, S. (2014). Competition as a way of life for $\mathrm{H}^{+}$-coupled antiporters. Journal of Molecular Biology, 426, 2539-2546. https://doi.org/10.1016/j.jmb.2014.05.020

Sigal, N., Molshanski-Mor, S., \& Bibi, E. (2006). No single irreplaceable acidic residues in the Escherichia coli secondary multidrug transporter MdfA. Journal of Bacteriology, 188, 5635-5639. https://doi.org/10.1128/JB.00422-06

Zhang, X. C., Zhao, Y., Heng, J., \& Jiang, D. (2015). Energy coupling mechanisms of MFS transporters. Protein Science, 24, 1560-1579. https://doi.org/10.1002/pro.2759

Cite this article: Law CJ (2021). Competition between protons and substrate for binding to the major facilitator superfamily multidrug/ $\mathrm{H}^{+}$antiporter MdtM Experimental Results, 2, e34, 1-9. https://doi.org/10.1017/exp.2021.26 


\section{Peer Reviews}

\section{Reviewing editor: Dr. Ioannis Pavlidis}

University of Crete, Rethimno, Greece, 74100

This article has been accepted because it is deemed to be scientifically sound, has the correct controls, has appropriate methodology and is statistically valid, and has been sent for additional statistical evaluation and met required revisions.

\section{doi:10.1017/exp.2021.26.pr1}

\section{Review 1: Competition between protons and substrate for binding to the major facilitator superfamily multidrug/ $\mathrm{H}^{+}$antiporter MdtM}

Reviewer: Chris Mulligan iD

University of Kent

Date of review: 23 August 2021

(C) The Author(s), 2021. Published by Cambridge University Press. This is an Open Access article, distributed under the terms of the Creative Commons Attribution licence (http://creativecommons.org/licenses/by/4.0), which permits unrestricted re-use, distribution and reproduction, provided the original article is properly cited.

Conflict of interest statement. Reviewer declares none.

Comments to the Author: This manuscript reports on the effects of $\mathrm{pH}$ on antimicrobial binding to the well characterised multidrug efflux pump, MdtM.

The experiment reported is well designed and executed to an excellent standard producing very clear results that are well interpreted. These data fully support previously published research on other protondriven drug efflux pumps suggesting competitive binding of protons is a common mechanism for proton-driven drug efflux pumps.

\section{Score Card}

Presentation

Is the article written in clear and proper English? (30\%)

Is the data presented in the most useful manner? (40\%)

Does the paper cite relevant and related articles appropriately? (30\%)

Context

Does the title suitably represent the article? (25\%)

Does the abstract correctly embody the content of the article? (25\%)

Does the introduction give appropriate context? (25\%)

Is the objective of the experiment clearly defined? (25\%) 
Are the limitations of the experiment as well as the contributions of the experiment clearly outlined? (20\%) 


\section{Review 2: Competition between protons and substrate for binding to the major facilitator superfamily multidrug/ $\mathrm{H}^{+}$antiporter MdtM}

Reviewer: Dr. Hiroshi Omote (iD

Okayama University

Date of review: 16 October 2021

(C) The Author(s), 2021. Published by Cambridge University Press. This is an Open Access article, distributed under the terms of the Creative Commons Attribution licence (http://creativecommons.org/licenses/by/4.0), which permits unrestricted re-use, distribution and reproduction, provided the original article is properly cited.

Conflict of interest statement. Reviewer declares none.

Comments to the Author: In this paper, author investigated $\mathrm{pH}$ dependence of substrate binding to MdtM multidrug transporter. Author measured quench of tryptophan fluorescence induced by TPP binding at various $\mathrm{pHs}$. Results clearly showed that TPP binding to MdtM is inhibited by acidic $\mathrm{pH}$ with apparent $\mathrm{pKa}$ of 6.2. This suggests importance of protonation/deprotonation process in substrate binding and proton/drug antiport.

Experiments are well designed. Results are interesting and clearly presented.

Few suggestions:

1) Please indicate counter ion for Bis-Tris propane buffer.

2) Please describe equations for calculation of Kdapp, Ki and $\mathrm{pKa}$.

3) It is better to add emission spectra of TPP bound form in figure 3.

4) Results are quite similar to those of MdfA except 10-fold different affinity. Does this difference correlate with apparent Km of TPP transport? If kdapps of MdfA and MdtM are correlated with Kms of these transporters, it supports the conclusion that $\mathrm{pH}$ dependent affinity change observed in this report is a part of transport process, and not an artifact.

5) Inhibition of TPP binding by protons can be discussed as protonation of specific acidic residues based on the mutagenic and structural analyses. If you have any idea, please discuss it.

\section{Score Card}

Presentation

5.0

Is the article written in clear and proper English? (30\%)

Is the data presented in the most useful manner? (40\%)

Does the paper cite relevant and related articles appropriately? (30\%)

Context

Does the abstract correctly embody the content of the article? (25\%)

Does the introduction give appropriate context? (25\%)

Is the objective of the experiment clearly defined? (25\%) 
Are the limitations of the experiment as well as the contributions of the experiment clearly outlined? (20\%) 